\title{
Measure Controllable Volumetric Mesh Parameterization
}

\author{
Kehua Su${ }^{\mathrm{a}}$, Wei Chen ${ }^{\mathrm{b}}, \mathrm{Na} \mathrm{Lei}^{\mathrm{c}, \mathrm{d}, *}$, Li Cui $^{\mathrm{e}}$, Jian Jiang ${ }^{\mathrm{b}}$, Xianfeng David Gu ${ }^{\mathrm{b}}$ \\ ${ }^{a}$ State Key Laboratory of Software Engineering, Wuhan University, Wuhan, 430072 China \\ ${ }^{b}$ Computer Science Department, Stony Brook University, Stony Brook, New York, 11794 USA \\ ${ }^{c}$ School of Software, Dalian University of Technology, Dalian, 116620 China \\ ${ }^{d}$ Yau Mathematics Science Center, Tsinghua University, Beijing, 100084 China \\ e School of Mathematical Science, Beijing Normal University, Beijing, 100875 China
}

\begin{abstract}
Volumetric parameterization is a fundamental problem in solid and physical modeling. In practice, it is highly desirable to control the volumes of the regions of interests in the parameter domain. This work introduces a novel volumetric parameterization method, which allows users to prescribe the target volumetric measure of the input solid.

Given a simply connected tetrahedral mesh with a single boundary surface, we first compute a volumetric harmonic map to parameterize the solid onto the unit solid ball; then we compute an optimal mass transportation map from the unit solid ball with the push-forward volume element induced by the harmonic map onto the parameter domain with the user prescribed volumetric measure. The composition of the volumetric harmonic map and the optimal mass transportation map gives a measure controllable volumetric parameterization. Furthermore, this method can handle solids with empty voids inside.

The method has solid theoretic foundation, and is based on conventional algorithms in computational geometry, and easy to implement. The experimental results demonstrate the efficiency and efficacy of the proposed method.
\end{abstract}

Keywords:

parameterization, volume, controllable, optimal mass transportation

\section{Introduction}

In solid and physical modeling, volumetric parameterization plays a fundamental role, it converts unstructured tetrahedron mesh to Spline surfaces/solids.

Volumetric parameterization is essential for applications in many engineering and medicine fields, such as Spline fitting in Computer Aided Design (CAD) [1], simulation in Computer Aided Engineering (CAE), volumetric meshing in digital geometry processing [2], volumetric texture mapping in Computer Graphics, volumetric medical image registration in medical imaging [3] and so on.

Volumetric mesh parameterization refers to the process of mapping a tetrahedral mesh onto a canonical domain in three dimensional Euclidean space $\mathbb{R}^{3}$. The mapping is required to be homeomorphic and minimizes geometric distortions. In practice, the user prefers to allocate greater volumes in the parameter domain for the regions of interests; or the solid consists of different materials, in order to meet the accuracy requirements in the simulation/analysis process, different materials need to allocate the knots with different resolutions, therefore different parametric volumes. These requirements motivate our measure controllable volumetric parameterization.

\footnotetext{
${ }^{*}$ Corresponding author.

Email addresses: skhemailg@gmail . com (Kehua Su), wei.chen. sbu@gmail.com (Wei Chen), nalei@outlook.com (Na Lei), licui@bnu. edu.cn (Li Cui), jianjiang@cs.stonybrook.edu (Jian Jiang), gu@cs.stonybrook. edu (Xianfeng David Gu)
}

Our method gives users the freedoms to assign a target measure to the input solid. Equivalently, the user can prescribe a positive Jacobian function on the whole target domain. So that the user can enlarge some regions and shrink the others, which is valuable for Spline fitting purpose. The regions with complicated geometries or materials can be mapped to large regions in the parameter domain, and obtain more knots and control points for Splines. This helps improve the robustness and accuracy for the down stream simulation/analysis. As a special case, our method can make the whole parameterization to be volumepreserving, this is advantageous for many applications in medical imaging and visualization. Volume-preserving parameteriztaion preserves the volumetric element, namely the Jacobian of the mapping equals to 1 everywhere.

In this work, we propose to use optimal mass transportation framework to achieve this goal. Given a convex domain $\Omega$ in the Euclidean space with two different measures (volume elements) $\mu$ and $v$, a Measure Preserving Map is an automorphism of the domain itself $\varphi: \Omega \rightarrow \Omega$, and preserves the measure. Namely, for any Borel set $B \subset \Omega$, the following equality holds

$$
\int_{\varphi^{-1}(B)} d \mu=\int_{B} d v
$$

The Quadratic Transportation Cost of the map $\varphi$ is given by

$$
C(\varphi):=\int_{\Omega}|p-\varphi(p)|^{2} d \mu(p) .
$$

The Optimal Mass Transportation Map is the measure- 
preserving map with the least quadratic transportation cost. Optimal mass transportation guarantees the existence and the uniqueness of the map.

According to Brenier's theorem, the optimal transportation map is the gradient map of a convex function defined on the domain. The problem of finding the optimal transportation map boils down to finding the convex function. In practice, the target measure is approximated by discrete measures (Dirac measures), the convex function is approximated by the upper envelope of a family of hyper-planes in $\mathbb{R}^{4}$, the normals of the planes are fixed but the heights (intercepts) are unknown. The heights can be obtained by optimizing a convex energy using Newton's method.

In our current work, for a given simply connected tetrahedral mesh with a single boundary surface, we first map it onto the unit solid ball using a harmonic map; then we compute an optimal mass transportation map of the unit ball from the user prescribed volume element to the canonical Euclidean volume element. The composition of the harmonic map and the optimal mass transportation map gives the measure-controllable parametreization of the initial tetrahedral mesh. Furthermore, this method can handle solids with empty voids inside.

Contributions. This work proposes a novel algorithm to compute volumetric parameterization with a prescribed measure for a simply connected tetrahedral mesh with a single boundary surface, furthermore the method is capable of handling solids with voids inside. The algorithm is based on the recently developed discrete optimal mass transportation theory [4], which is rigorous and easy to implement. This work mainly focuses on the algorithmic design and implementation.

\section{Previous Works}

The literature for parametreization is vast, so a thorough survey is beyond the scope of the current work. In the following, we only briefly review the most related works in volumetric parameterization and optimal mass transportation.

\subsection{Volumetric Parameterization}

There are mainly three kinds of methods for volumetric parameterization.

The most widely used method is harmonic mapping, whose basic idea is to reduce the discrete harmonic energy by a variational procedure. This method was first proposed by Wang et al. in [3]. They mapped a genus-zero volume to a solid sphere, and later they used this method on brain mapping [5]. The harmonic mapping between two solid models was computed with a meshless approach by Li et al. [6]. Martin et al. constructed trivariate spline for cylindrical volumes by computing harmonic volumetric parameterization in [7]. Xu et al. [8] designed a bi-harmonic map applying a multiple fundamental solutions system for fast computation. Using Green's functions, Xia et al.[9] parameterized star-shaped volumes. They showed that the constructed map is bijective and smooth except at unique critical point. They also proposed an algorithm to decompose a volume into the direct product of a two-dimensional (2D) surface and a one-dimensional (1D) curve and then traced the integral curve along the harmonic function in [10]. In 2014, by combining harmonic map and streamline approach, Gupta et al. [11] presented an approach for the problem of volumetric parameterization of a general nonconvex (genus-0) domain to its topologically equivalent convex domain.

Many mappings are constructed by means of generalized barycentric coordinates with closed form expressions [12]. The mean-value coordinates method was extended from surface [13] to volume by $\mathrm{Ju}$ et al. [14] and Floater et al. [15] by computing the interpolation of volumetric data. Lipman et al. [16] proposed Green coordinates which lead to mappings with shape-preserving property.

Another kind of popular method is to find a mapping minimizing a specific energy. Chao et al.[17] minimized the socalled ARAP (as-rigid-as-possible) deformation energy, which is a simple geometric model measuring distance from the Jacobian of the mapping to an isometry. Frame field driven methods use the energy measuring difference between the Jacobian and the guidance frame field [18][19][20] and compute the volumetric parameterization in a variational way. In 2015, by deriving a 3D version of stretch-distortion energy and incorporating fixed boundary conditions, Jin et al. [21] extended the stretchminimizing method to volumetric parameterization.

\subsection{Optimal Mass Transportation}

Monge raised the classical Optimal Mass Transport Problem that concerns determining the optimal way, with minimal transportation cost, to move a pile of soil from one place to another [22]. Kantorovich [23] has proved the existence and uniqueness of the optimal transport plan based on linear programming. Monge-Kantorovich optimization has been used in numerous fields from physics, econometrics to computer science including data compression and image processing [24]. Recently, researchers have realized that optimal transport could provide a powerful tool in image processing, if one could reduce its high computational cost $[25,26]$. However, it has one fundamental disadvantage that the number of variables is $O\left(k^{2}\right)$, where $k$ is the number of discrete samples, which is unacceptable to computer vision and medical imaging applications since a high resolution 3D surface normally includes up to hundreds of thousands of vertices.

An alternative Monge-Brenier optimization scheme can significantly reduce the number of variables to be optimized. In late 1980's, Brenier [27] developed a different approach for a special class of optimal transport problems, where the cost function is a quadratic distance. Brenier's theory shows that the optimal transport map is the gradient map of a special convex function. Assume the target domain is discretized to $k$ samples, the Monge-Brenier's approach reduces the unknown variables from $O\left(k^{2}\right)$ to $O(k)$, which greatly reduces the computation cost, and improves the efficiency. In our framework, we take Monge-Brenier's approach. However, our work is based on the newly discovered variational principle [4] which is the underspinning of Monge-Brenier's approach. Our framework is general and works with any valid measures, $\mu$ and $v$, defined on 
two surfaces. Within the scope of this paper, we only consider the area induced measures. Recently, Su et al. applied Brenier's approach for shape matching and comparison in computer vision field [28]. Similar method has been used for surface area-preserving parameterization in graphics/visualization field by Kaufman et al. in [29], which focuses on the optimal mass transportation maps between 2D surfaces. This work focuses on the mappings between $3 D$ volumetric domains, which involves not only more sophisticated data structures and algorithms, but also more theoretic challenges. A harmonic map from a simply connected surface with a single boundary to a convex planar domain is diffeomorphic if the restriction on the boundary is homeomorphic, but it is widely open that when a volumetric harmonic map is diffeomorphic. In theory, this makes volumetric parameterization intrinsically much more difficult. So far, to the best of the authors' knowledge, there is no existing work on volume-preserving parameterization.

\section{Theoretic Background}

This section briefly introduces the theoretic background of Optimal Mass Transport theory. We refer readers to [23] for Kantorovich's approach, [27] and [30] for Breniner's approach, [4] for more detailed proofs of the proposed method.

\subsection{Optimal Mass Transport}

Monge [22] raised the optimal mass transportation problem in the 18 th century.

Problem 3.1 (Optimal Mass Transport). Suppose $(X, \mu),(Y, v)$ are metric spaces with probabilities measures, which have the same total mass $\mu(X)=v(Y)$. A map $T: X \rightarrow Y$ is measure preserving, if for any measurable set $B \subset Y, \mu\left(T^{-1}(B)\right)=\nu(B)$. Given a transportation cost function $c: X \times Y \rightarrow \mathbb{R}$, find the measure preserving map $T: X \rightarrow Y$ that minimizes the total transportation cost

$$
C(T):=\int_{X} c(x, T(x)) d \mu(x) .
$$

In the 1940's, Kantorovich introduced the relaxation of Monge's problem and solved it using linear programming method [23]. At the end of 1980's, Brenier [27] discovered the intrinsic connection between optimal mass transport map and convex geometry.

Definition 3.1 (Convex Function). Suppose $f: X \rightarrow \mathbb{R}$ is a function, $f$ is convex if $f\left(\frac{x_{1}+x_{2}}{2}\right) \leq \frac{1}{2}\left(f\left(x_{1}\right)+f\left(x_{2}\right)\right)$. If $f$ is $C^{2}$ continuous convex function, its Hessian matrix is semi-positive definite. $\left(\frac{\partial^{2} f}{\partial x_{i} \partial x_{j}}\right) \geq 0$.

Suppose $f: X \rightarrow \mathbb{R}$ is a function, the gradient map $\nabla f:$ $X \rightarrow Y$ is defined as $x \mapsto \nabla f(x)$.

Theorem 3.1 (Brenier). Suppose $X$ and $Y$ are the Euclidean space $\mathbb{R}^{n}$, and the transportation cost is the quadratic Euclidean distance $c(x, y)=|x-y|^{2}$. If $\mu$ is absolutely continuous and $\mu$ and $v$ have finite second order moments, then there exists a convex function $f: X \rightarrow \mathbb{R}$, its gradient map $\nabla f$ gives the solution to the Monge's problem. Furthermore, the optimal mass transportation map is unique.

This theorem converts the Monge's problem to solving the following Monge-Amperè partial differential equation:

$$
\operatorname{det}\left(\frac{\partial^{2} f(x)}{\partial x_{i} \partial x_{j}}\right)=\frac{\mu(x)}{\nu \circ \nabla f(x)},
$$

Proof. By abusing symbols, $\mu$ and $v$ represent the measure densities. Use the change of variable formula on a small set $B \subset Y$ : $\int_{B} v(y) d y=\int_{T^{-1}(B)} \mu(x) d x=\int_{B}\left\|J_{T}(x)\right\| v(T(x)) d x$ injecting $T(x)=\nabla f(x)$, we get: $\int_{B} \mu(x) d x=\int_{B}\left\|\operatorname{Hess}_{f}(x)\right\| v(\nabla f(x)) d x$ Since the previous equation is true for all $\mathrm{B}$, we have $\mu(x)=$ $\left\|\operatorname{Hess}_{f}(x)\right\| v(\nabla f(x))$.

Detailed proof can be found in [31].

\subsection{Discrete Optimal Mass Transport}

Suppose $\mu$ has compact support on $X$, define $\Omega=\operatorname{supp} \mu=$ $\{x \in X \mid \mu(x)>0\}$, assume $\Omega$ is a convex domain in $X$. The space $Y$ is discretized to $Y=\left\{y_{1}, y_{2}, \cdots, y_{k}\right\}$ with Dirac measure $v=\sum_{j=1}^{k} v_{j} \delta\left(y-y_{j}\right)$.

The height vector is defined as $\mathbf{h}=\left(h_{1}, h_{2}, \cdots, h_{k}\right) \in \mathbb{R}^{k}$. Each $y_{i} \in Y$ corresponds to a hyperplane $\pi_{i}(\mathbf{h}):\left\langle x, y_{i}\right\rangle+h_{i}=0$. The piecewise linear convex function is given by

$$
u_{\mathbf{h}}(x)=\max _{i=1}^{k}\left\{\left\langle x, y_{i}\right\rangle+h_{i}\right\}
$$

The graph of $u_{\mathbf{h}}(x)$ is $G(\mathbf{h})$, which is an infinite convex polyhedron with supporting planes $\pi_{i}(\mathbf{h})$. The projection of $G(\mathbf{h})$ induces a polygonal partition of $\Omega, \Omega=\bigcup_{i=1}^{k} W_{i}(\mathbf{h})$, where each cell $W_{i}(\mathbf{h})$ is the projection of a facet of $G(\mathbf{h})$. The gradient map $\nabla u_{\mathbf{h}}: W_{i}(\mathbf{h}) \rightarrow y_{i}$ maps each $W_{i}(\mathbf{h})$ to a single point $y_{i}$. The area of $W_{i}(\mathbf{h})$ is denoted as $w_{i}(\mathbf{h})=\mu\left(W_{i}(\mathbf{h})\right)$.

The following theorem plays a fundamental role for discrete optimal mass transport theory.

Theorem 3.2. For any given measure $v$, such that $\sum_{j=1}^{n} v_{j}=\mu(\Omega), v_{j}>0$, there must exist a height vector $\mathbf{h} u$ nique up to adding a constant vector $(c, c, \cdots, c)$, the gradient map of the convex function $u_{\mathbf{h}}$ is measure-preserving $w_{i}(\mathbf{h})=v_{i}$, and optimizes the quadratic transportation cost.

The existence and uniqueness was first proven by Alexandrov [32] using a topological method, which cannot be converted to a computational algorithm; the regularity of the solution was given by Pogorelov in [33]; Aurenhammer, Hoffman and Aranov proved the theorem using power voronoi diagram in [34] and [35], which leads to constructive algorithm based on gradient descend and quasi-Newton method. Merigot et al. obtained the similar results in [36] and proposed hierarchical computational method in [37]. Brenier proved polar factorization in [27] using probability measures, his theory encompasses both the continuous, semi-discrete and discrete settings. Recently, $\mathrm{Gu}$ et al. [4] gave a novel proof for the existence and uniqueness based on the variational principle, which formulates the 
optimal mass transportation problem as a convex optimization, the Hessian of the energy has explicit geometric meanings.

Define the admissible space of height vectors $H_{0}:=$ $\left\{\mathbf{h} \mid \sum_{j=1}^{k} h_{j}=0\right.$ and $\left.\mu\left(W_{i}(\mathbf{h})\right)>0, \forall i=1, \cdots, k,\right\}$. Then define the energy $E(\mathbf{h})$,

$$
E(\mathbf{h})=\int_{\mathbf{0}}^{\mathbf{h}} \sum_{i=1}^{k} w_{i}(\eta) d \eta_{i}-\sum_{i=1}^{k} v_{i} h_{i} .
$$

The following theorem lays down the theoretic foundation of our OMT map algorithm.

Theorem 3.3 (Discrete Optimal Mass Transport [4]). If $\Omega$ is convex, then the admissible space $H_{0}$ is convex, the energy ( $E$ $q n .3)$ is convex. The unique global minimum $\mathbf{h}^{*}$ is an interior point of $H_{0}$. Furthermore, the gradient map of $u_{\mathbf{h}^{*}}$ is the unique optimal mass transport map.

The proof of Theorem 3.3 is reported in [4]. With this theory, the global minimum can be obtained efficiently using Newton's method due to the convexity of the energy. Comparing to Kantorovich's approach, where there are $O\left(k^{2}\right)$ unknowns, this approach has only $O(k)$ unknowns.

\section{Computational Algorithms}

This section focuses on algorithms. The theoretic deduction for optimal mass transportation map can be found in [28] and [4]. In order to be complete, we give all details of these algorithms.

\subsection{Discrete Optimal Mass Transportation Map}

Given a discrete point set $P \subset \mathbb{R}^{3}, P=\left\{\left(p_{i}, A_{i}\right), 1 \leq i \leq k\right\}$, such that $\sum_{i=1}^{k} A_{i}=1$, Our goal is to find a discrete optimal mass tansportation from the unit solid cube to the measured point set $P$, denoted as $\varphi: \mathbb{D}^{3} \rightarrow P$. The algorithm pipeline is summarized in Alg.1.

Brenier Potential. According to Brenier's theorem, there should be a convex function, the so-called Brenier potential $f: \mathbb{D}^{3} \rightarrow \mathbb{R}$, the optimal mass transportation map is given by the gradient map of the Brenier potential.

In the discrete setting, the Brenier potential is a piecewise linear convex function, constructed as follows. For each vertex $u \in M$, suppose $\mathbf{f}(u)$ is $\left(a_{u}, b_{u}, c_{u}\right)$, we construct a plane $\pi_{u}$ in the four dimensional Euclidean space $\mathbb{R}^{4}$, namely a linear function

$$
\pi_{u}(x, y, z)=a_{u} x+b_{u} y+c_{u} z+h_{u} .
$$

The Brenier potential is defined as

$$
f(x, y, z):=\max _{u \in M} \pi_{u}(x, y, z)=\max _{u \in M}\left\{a_{u} x+b_{u} y+c_{u} z+h_{u}\right\} .
$$

Its graph is the upper envelope of family of planes $\left\{\pi_{u}, u \in M\right\}$ in $\mathbb{R}^{4}$

We use $\Omega(\mathbf{h})$ to represent the (open) convex polyhedron of the upper envelope, where $\mathbf{h}=\left(h_{u}\right)$ is the height vector. $\pi_{u}$ 's are the supporting planes of the upper envelope $\Omega(\mathbf{h})$, the face $F_{u}$ is the intersection between $\pi_{u}$ and $\Omega(\mathbf{h})$,

$$
F_{u}=\Omega(\mathbf{h}) \cap \pi_{u},
$$

the projection of $F_{u}$ into $\mathbb{R}^{3}$ is defined as a cell $W_{u}$ in $\mathbb{R}^{3}$,

$$
W_{u}(\mathbf{h}):=\left\{(x, y, z) \in \mathbb{R}^{3} \mid \nabla f(x, y, z)=\left(a_{u}, b_{u}, c_{u}\right)\right\} .
$$

Therefore, the projection of the upper envelope $\Omega(\mathbf{h})$ to $\mathbb{R}^{3}$ induces a cell decomposition of the unit solid cube, denoted as $\mathcal{D}(\mathbf{h})$ :

$$
\mathbb{D}^{3}=\bigcup_{u \in M} \mathbb{D}^{3} \cap W_{u}(\mathbf{h}),
$$

This cell decomposition is called as the power Voronoi diagram of $\mathbb{D}^{3}$ [38]. The volume of each cell is defined as

$$
w_{u}(\mathbf{h})=\operatorname{vol}\left(\mathbb{D}^{3} \cap W_{u}(\mathbf{h})\right) .
$$

Legendre Dual. The computation of the upper envelope of a family of planes $\left\{\pi_{u}, u \in M\right\}$ is converted to convex construction of its Legendre dual.

Definition 4.1 (Legendre Dual). Suppose $f: \mathbb{R}^{3} \rightarrow \mathbb{R}$, its Legendre dual is a function $f^{*}: \mathbb{R}^{3} \rightarrow \mathbb{R}$, defined as

$$
f^{*}\left(x^{*}, y^{*}, z^{*}\right):=\sup _{(x, y, z) \in \mathbb{R}^{3}}\left\{x x^{*}+y y^{*}+z z^{*}-f(x, y, z)\right\} .
$$

Each plane $\pi_{u}(x, y, z)=a_{u} x+b_{u} y+c_{u} z+h_{u}$ is dual to a point

$$
\pi_{u}^{*}:=\left(a_{u}, b_{u}, c_{u},-h_{u}\right) \in \mathbb{R}^{4} .
$$

The upper envelope of the supporting planes

$$
\Omega(\mathbf{h})=\operatorname{Env}\left(\left\{\pi_{u}, u \in M\right\}\right)
$$

is the graph of the Brenier potential $f$; the graph of the Legendre dual of the Brenier potential $f^{*}$ is the lower convex hull of the dual points of the supporting planes

$$
\Omega^{*}(\mathbf{h}):=\operatorname{Conv}\left(\left\{\pi_{u}^{*}, u \in M\right\}\right) .
$$

The projection of the lower convex hull $\Omega^{*}(\mathbf{h})$ to $\mathbb{R}^{3}$ forms a triangulation, which we call as the power Delaunay triangulation, denoted as $\mathcal{T}(\mathbf{h})$.

The dual relations between the power Voronoi diagram $\mathcal{D}(\mathbf{h})$ and the power Delaunay triangulation $\mathcal{T}(\mathbf{h})$ can be represented as follows: each cell $W_{u}(\mathbf{h}) \subset \mathcal{D}(\mathbf{h})$ is dual to the vertex $\mathbf{f}(u) \subset$ $\mathcal{T}(\mathbf{h})$; the 3 dimensional cell $W_{u}(\mathbf{h}) \cap W_{v}(\mathbf{h}) \subset \mathcal{D}(\mathbf{h})$ is dual to the edge $\{\mathbf{f}(u), \mathbf{f}(v)\} \subset \mathcal{T}(\mathbf{h})$; each vertex in the Voronoi diagram is the intersection of 4 cells $W_{u}(\mathbf{h}) \cap W_{v}(\mathbf{h}) \cap W_{w}(\mathbf{h}) \cap W_{l}(\mathbf{h}) \subset$ $\mathcal{D}(\mathbf{h})$ corresponds to a tetrahedron in the Delaunay triangulation $\{\mathbf{f}(u), \mathbf{f}(v), \mathbf{f}(w), \mathbf{f}(l)\} \subset \mathcal{T}(\mathbf{h})$.

Optimization. The key to find the optimal mass transportation map is to find the appropriate height vector $\mathbf{h}$, then construct the upper envelope $\Omega(\mathbf{h})$, and the Brenier potential function, whose gradient map is the desired map. The height vector is the unique minimizer of the following convex energy

$$
E(\mathbf{h})=\int^{\mathbf{h}} \sum_{u \in M} w_{u}(\eta) d \eta_{u}-\sum_{u \in M} A_{u} h_{u},
$$


with the constraint

$$
\sum_{u \in M} A_{u} h_{u}=0
$$

Due to the convexity of the energy, it can be optimized using Newton's method. The gradient of the energy has the form

$$
\nabla E(\mathbf{h})=\left(w_{u}(\mathbf{h})-A_{u}\right), u \in M
$$

The Hessian matrix of the energy can be constructed geometrically, which involves the 2-cells of the power Voronoi diagram and their dual edges in the power Delaunay triangulation. In details, if the cells $W_{u}(\mathbf{h})$ and $W_{v}(\mathbf{h})$ are adjacent in the power Voronoi diagram $\mathcal{D}(\mathbf{h})$, then their intersection is a 2-cell $W_{u}(\mathbf{h}) \cap W_{v}(\mathbf{h})$, the dual of this 2-cell in the power Delaunay triangulation $\mathcal{T}(\mathbf{h})$ is an edge $\{\mathbf{f}(u), \mathbf{f}(v)\}$, then we define

$$
k_{u, v}:=\frac{\operatorname{Area}\left(W_{u}(\mathbf{h}) \cap W_{v}(\mathbf{h})\right)}{|\mathbf{f}(u)-\mathbf{f}(v)|} .
$$

If $W_{u}(\mathbf{h}) \cap W_{v}(\mathbf{h})=\emptyset$, then the corresponding $k_{u, v}$ is 0 . The Hessian matrix is given by

$$
\frac{\partial^{2} E(\mathbf{h})}{\partial h_{u} \partial h_{v}}:= \begin{cases}-k_{u, v} & u \neq v \\ \sum_{w} k_{u, w} & u=v\end{cases}
$$

At the first step, we initialize the height vector as

$$
h_{u}=-\frac{1}{2}\langle\mathbf{f}(u), \mathbf{f}(u)\rangle=-\frac{1}{2}\left(a_{u}^{2}+b_{u}^{2}+c_{u}^{2}\right)
$$

At each step, we solve the linear system using the conjugate gradient solver provided by Eigen [39],

$$
\left(\frac{\partial^{2} E(\mathbf{h})}{\partial h_{u} \partial h_{v}}\right) \delta \mathbf{h}=\nabla E(\mathbf{h})
$$

then update the height vector $\mathbf{h} \leftarrow \mathbf{h}-\alpha \delta \mathbf{h}$, until the norm of the gradient $\nabla E(\mathbf{h})$ is less than a predefined threshold. The step length $\alpha$ is controlled using the damping method similar to that in [40]. We choose the smallest integer $k \in \mathbb{Z}$, such that $\mathbf{h}-2^{-k} \delta \mathbf{h}$ in admissible, (namely in $H_{0}$ ), then set the step length $\alpha$ as $2^{-k}$.

\subsection{Volumetric Parameterization with Prescribed Measures}

Let $M$ be the simply connected tetrahedral mesh with a single boundary surface, we would like to compute a measure controllable mapping $M$ to the unit solid cube $\mathbb{D}^{3}$. The algorithm pipeline is described in Alg. 2.

First, we scale the solid, such that its total volume is equals to that of the unit solid cube. We use the algorithms described in [3] to find a volumetric harmonic map from $M$ to $\mathbb{D}^{3}, \mathbf{f}: M \rightarrow$ $\mathbb{D}^{3}$.

Second, we decide the target measure $A_{u}$ for each vertex $u \in$ $M$. The target measure could be assigned by the user, as long as the the total measure equals the volume of the unit solid cube, $\sum_{u \in M} A_{u}=1$. Otherwise, we can use the push-forward measure induced by the harmonic map. For each vertex $u \in M$, we

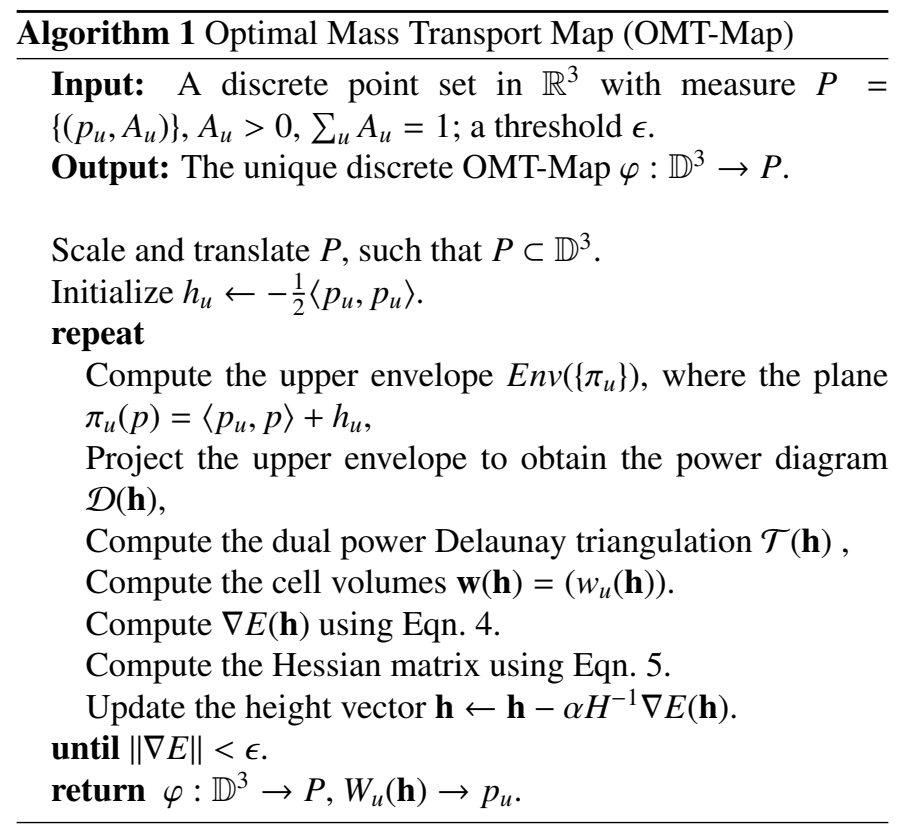

define the measure associated with it as the one fourth the total volume of all tetrahedra adjacent to it,

$$
A_{u}:=\frac{1}{4} \sum_{\{u, v, w, l\} \in M} \operatorname{vol}(\{u, v, w, l\}),
$$

then we define the discrete point set with measures

$$
P=\bigcup_{u \in M}\left\{\left(\mathbf{f}(u), A_{u}\right)\right\}
$$

Finally, we compute the discrete optimal mass transportation map $\varphi: \mathbb{D}^{3} \rightarrow P$. The inverse map $\varphi^{-1}$ maps each point $p_{u} \in P$ to the mass center of $W_{u}$. The composition $\varphi^{-1} \circ \mathbf{f}: M \rightarrow \mathbb{D}^{3}$ is the desired volume-preserving parameterization.

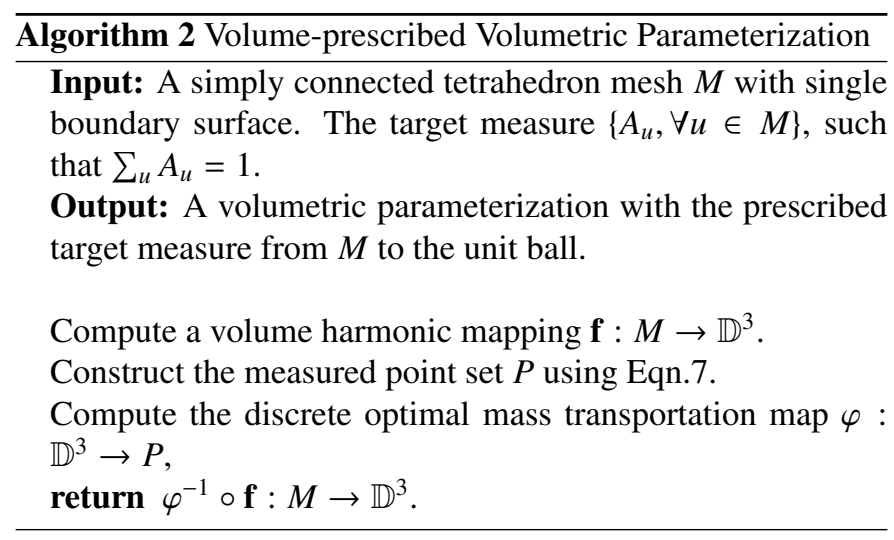

This method can be directly generalized to handle solids with voids inside. If the input solid $M$ has multiple boundaries, $\partial M=S_{0}-S_{1} \cdots-S_{k}$, where $S_{0}$ is the exterior boundary surface and $S_{i}$ 's are interior boundary surfaces for $i>0$. We fill each interior boundary surface $S_{i}$ by a solid $\Omega_{i}, \partial \Omega_{i}=S_{i}$. We use the Euclidean volumetric element as the measures defined on $\Omega_{i}$ 's. Then we compute the volume-preserving map from the 
solid with voids filled to the unit solid ball, $\varphi: M \bigcup_{i} \Omega_{i} \rightarrow \mathbb{D}^{3}$, then the restriction of the mapping

$$
\left.\varphi\right|_{M}: M \rightarrow \mathbb{D}^{3} \backslash \bigcup_{i=1}^{k} \varphi\left(\Omega_{i}\right)
$$

gives the volume-preserving parameterization of $M$.

\section{Experimental Results}

In this section, we demonstrate the efficiency and efficacy of our method using examples from the real world. All the experiments are conducted on a laptop computer of Intel Core i54200U CPU, 2.29GHz with 8GB memory. All the algorithms are implemented using generic $\mathrm{C}++$. The Power Voronoi diagram are computed using CGAL [41]. The linear systems are solved using numerical package Eigen [39].

\subsection{Volume-preserving Parameterization}

We have tested our volumetric parameterization on several models, including Michelangelo's King David Head, Stanford Bunny, the Bimba sculpture, the Buddha model, the Duck model and the Lion Cup model. All results are shown in Figure 1. Row (a) shows the input solid tetrahedron mesh; Row (b) shows 3D boundary surfaces; Row (c) illustrates the spherical conformal mapping result of (b). We calculate the volumetric harmonic mapping using the Dirichlet boundary condition of (b). The Optimal Mass Transporation Map (OMT) results are shown in Row (d), (e) and (f), with exterior view, cutaway view and side view respectively.

Our method can be extended directly to the solids with voids, as shown in Fig 2. Frame (a) and (b) show the original volumetric models, a solid torus is removed from the Lion Cup model and a solid ball is removed from the Stanford bunny model; Frame (c) and (d) are the volume preserving parameterization results.

\subsection{Volumetric Morphing}

Volume-preserving parameterization can be directly applied to volumetric morphing. Using volumetric harmonic mapping and OMT technique, we can transform one complex solid model to the canonical cube. Figure 3 and 4 show the animation sequence of volumetric morphing, using our volumetricpreserving parameterization technique.

\subsection{Importance Driven Volume Parameterization}

Our method allows users to fully control the volume of regions of interests (ROI). By adjusting the target measure, the user can enlarge or shrink specific regions by a scaling factor $S$ as shown in Figure 7 and 8. In Figure 7, the ROI is a single region. Furthermore, we can also control the measure for multiple ROIs shown in Figure 8.

\subsection{Comparison}

We have compared our volume-preserving parameterization technique with the method in [42] where the authors propose to utilize the uniform sampling and the hierarchy algorithm to accelerate the computation of OMT. However, this method changes the tetrahedron structure of the input solid, only giving an approximation to OMT. Additionally, the final result is not bijective on the boundary. In contrast, our method preserves the combinatorial structure of the input solid, giving the accurate solution and the result is bijective for both interior and the boundary surface. Therefore, our method can produce smoother and more physically sensible morphing sequence. By comparing the morphing sequences generated by our method in Figure 3 and 4 and those by the method of [42] in Figure 5 and 6 , we can see the other method produces large foldings near the boundary surfaces, whereas our method gives diffeomorphic deformation for the whole solids including the boundaries.

\subsection{Quantitative Analysis}

In order to verify the volume-preserving property of our proposed parameterization algorithm, we calculate the histograms of angle distortion and volume distortion explicitly. For volume distortion, we compute the ratio between the volume of each tetrahedron in the source solid and the volume of its image, and illustrate the histograms of the logarithms of the volume ratios. For angle distortion, we compute the ratio between each dihedral angle in the source solid and that of its image in the parameter domain, and show the histograms of the logarithms of the dihedral angle-ratios.

Figure 10 shows the distortion distributions of the King David's Head, the Stanford Bunny, the Buddha, the Bimba, the Duck and the Lion Cup model, respectively. For each model, Column (1) shows the volume distortion of the volumetric harmonic mapping; Column (2) illustrates the volume distortion of the volume-preserving parameterization; Column (3) is the angle-distortion of the harmonic mapping; Column (4) is the angle-distortion of the volumetric-preserving parameterization.

By carefully examining histograms in Row (a) and (b), one can see that the volume distortion histograms produced by the volume-preserving parameterization algorithm are highly concentrated near the origin, which verifies that our parameterizations preserve the volume elements very well; in contrast, the volume-distortion histograms produced by the volumetric harmonic mappings are much more dispersed. The angledistortion histograms in Row (c) and (d) show that our volumepreserving parameterization method produces small angle distortions as well.

Table 1 summarizes the number of iterations and the computation time for different models. Current method doesn't apply any sophisticated optimization, such as hierarchy computation and dynamic kinetics. In average, the number of iterations of gradient descent method is tens of times more than that of Newton's method, the running time of the former is tens of times longer than that of the latter. Unlike the surface harmonic mapping, the volumetric harmonic mapping cannot guarantee the mapping to be bijective. In general, there will be small number of flipped tetrahedra, which can be easily fixed manually. 
(a)
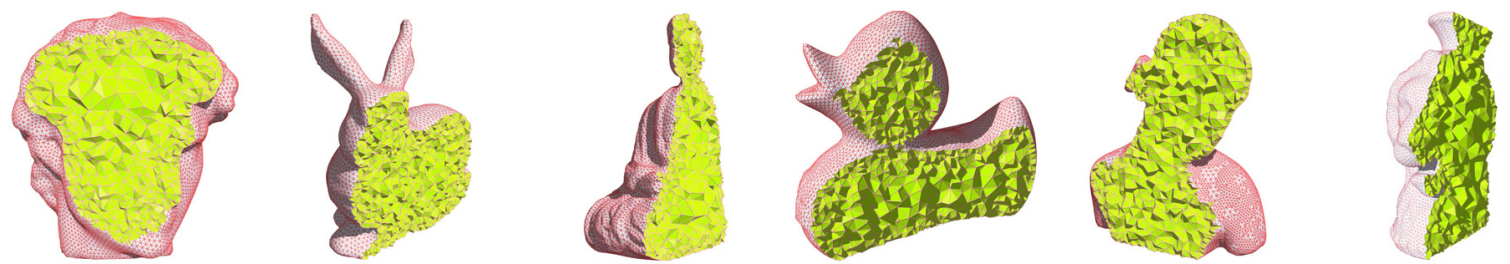

(b)
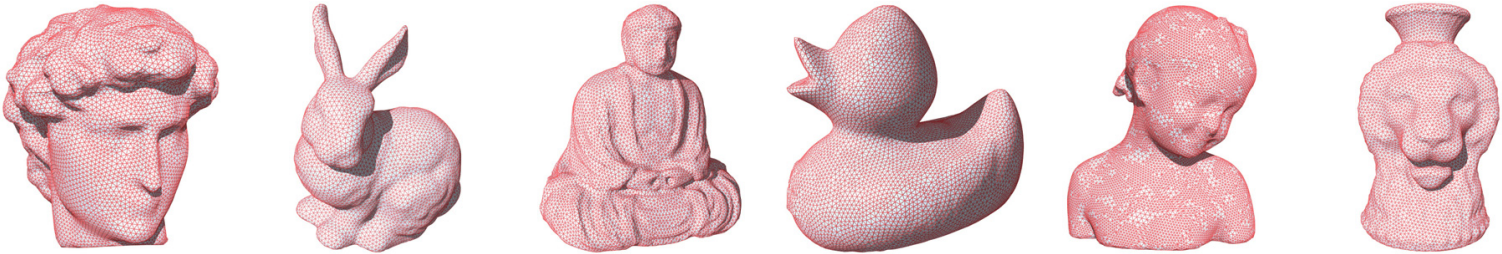

(c)
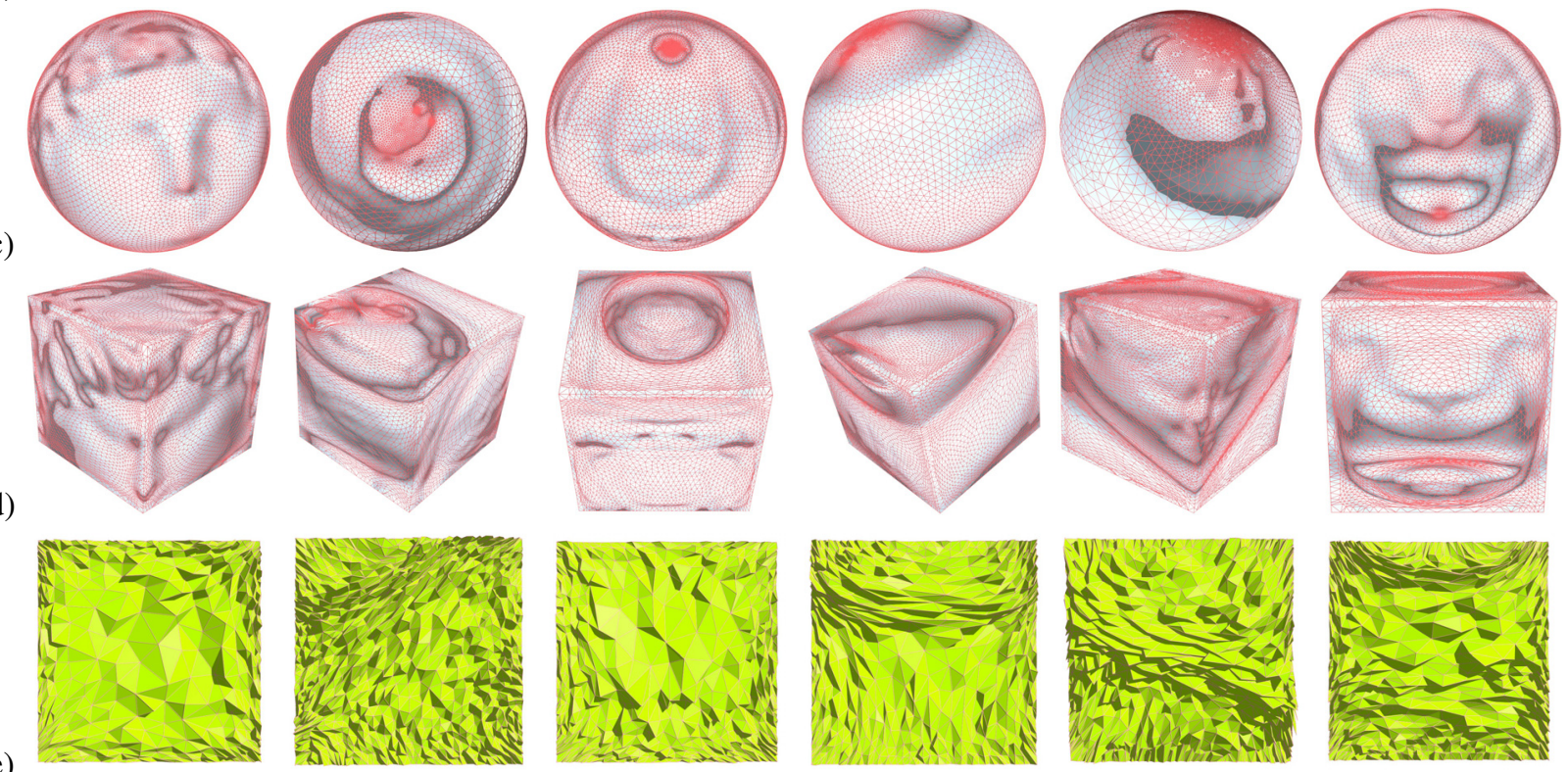

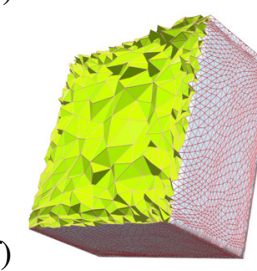

(1)

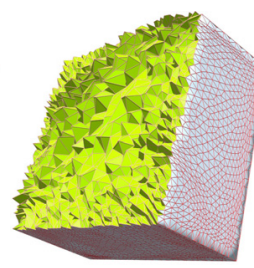

(2)

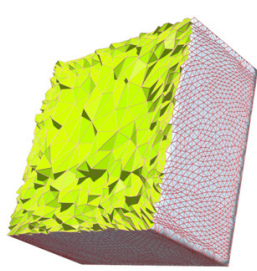

(3)

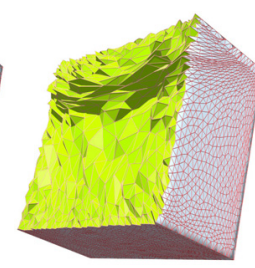

(4)

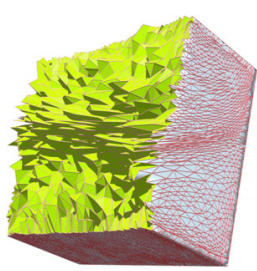

(5)

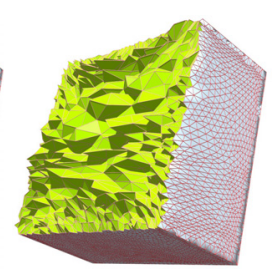

(6)

Figure 1: Volume-preserving parameterization for different volumetric meshes. Column (1)-(6) shows the model of the King David Head, the Stanford Bunny, the Buddha, the Duck, the Bimba and the Lion Cup, respectively. Row (a) shows the volumetric meshes. (b) illustrates the boundary surfaces. (c) is the spherical conformal map of (b). (d)(e) and (f) are OMT results. 


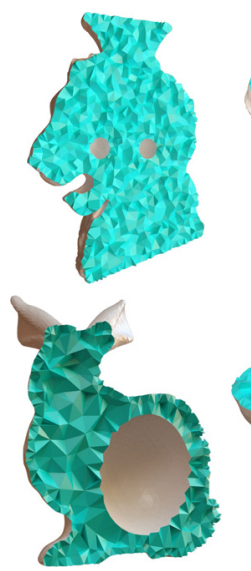

(a)
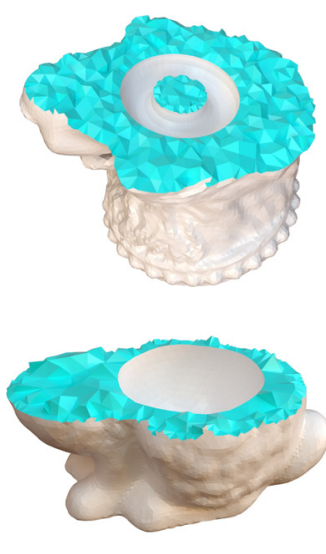

(b)
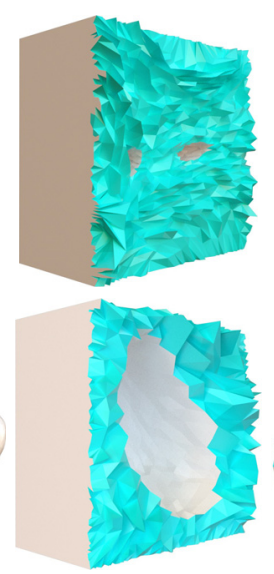

(c)

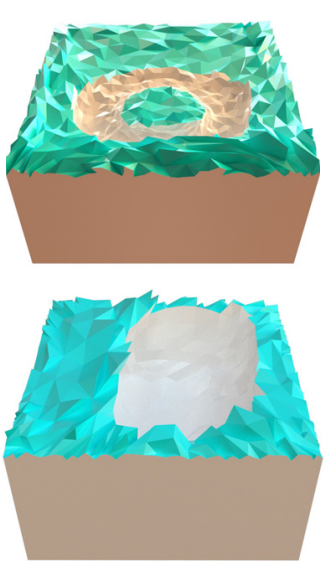

(d)

Figure 2: Volume preserving parameterization for solids with voids. A solid torus and a solid ball are removed from the Lion Cup model and the Stanford Bunny model respectively, which are shown in the top and bottom row. Column (a) and (b) show the solids with voids from different views. Column (c) and (d) show the OMT results.
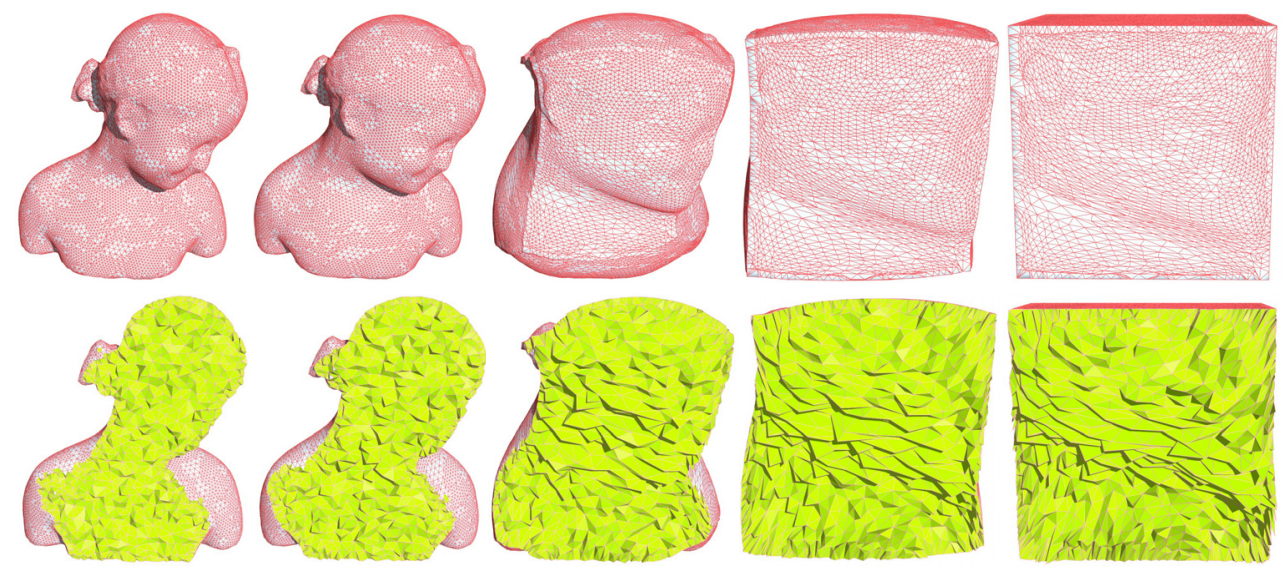

(a) $t=0$

(b) $t=0.25$

(c) $t=0.5$

(d) $t=0.75$

(e) $t=1.0$

Figure 3: The volumetric morphing process from the Bimba sculpture to the canonical cube using our volumetric-preserving parameterization technique. $t$ is the time parameter in the morphing process.
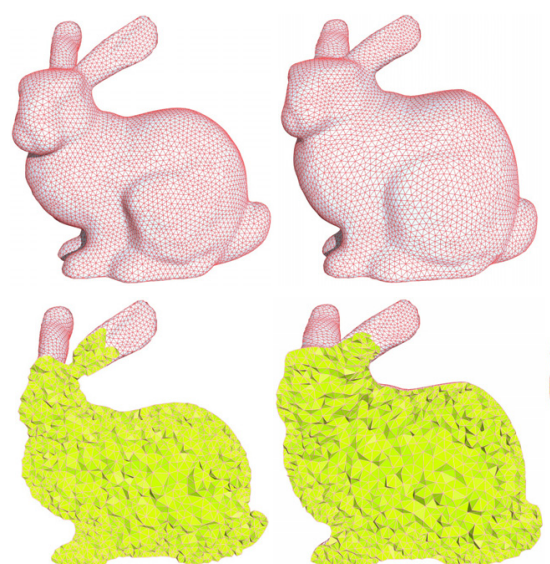

(a) $t=0$

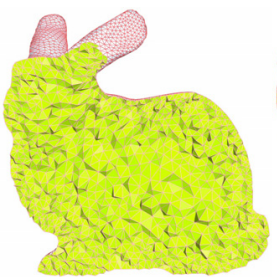

(b) $t=0.25$
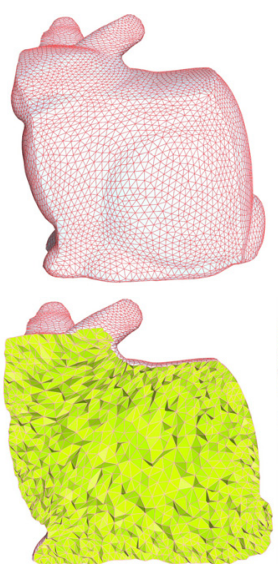

(c) $t=0.5$
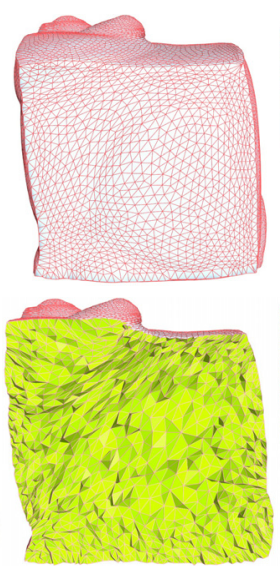

(d) $t=0.75$
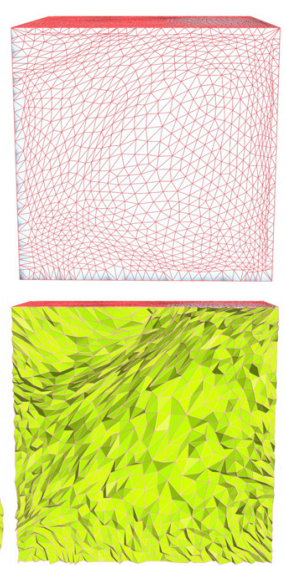

(e) $t=1.0$

Figure 4: The volumetric morphing process from the Stanford bunny to the canonical cube using our volumetric-preserving parameterization technique. $t$ is the time parameter in the morphing process. 


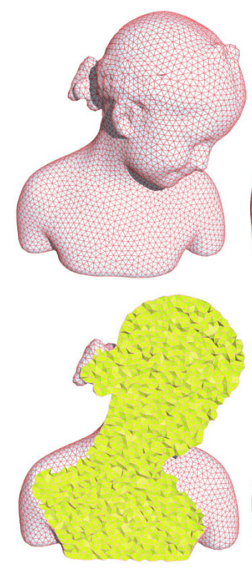

(a) $t=0$
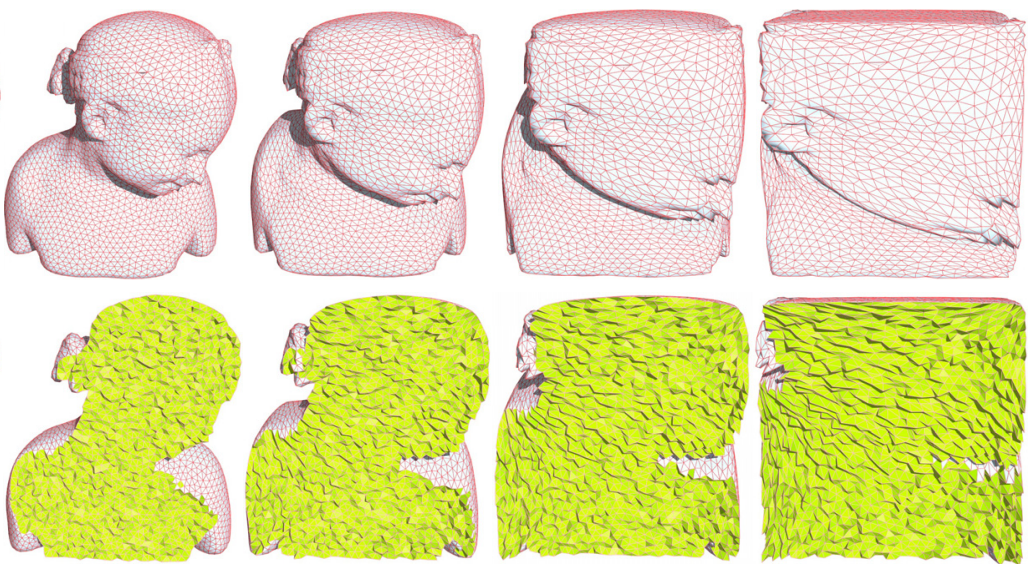

(d) $t=0.75$

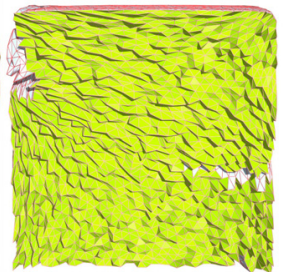

(e) $t=1.0$

Figure 5: The volumetric morphing sequence of the Bimba model (front view) using the method in [42]. $t$ is the time parameter in the morphing process.
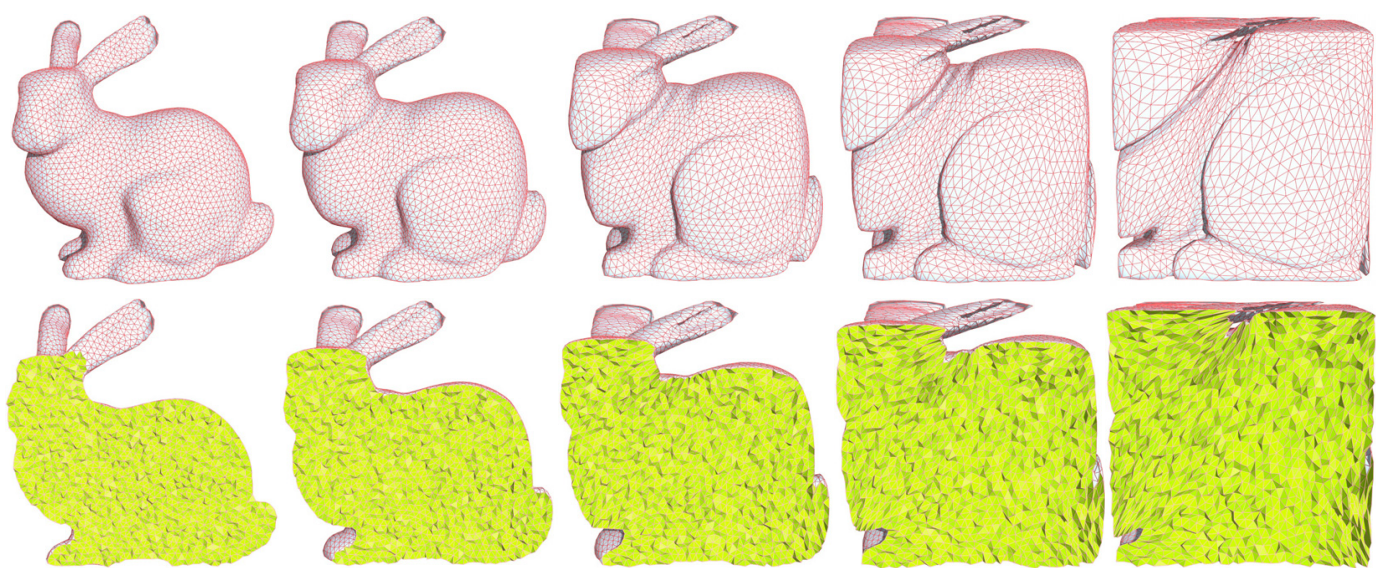

(a) $t=0$

(b) $t=0.25$

(c) $t=0.5$

(d) $t=0.75$

(e) $t=1.0$

Figure 6: The volumetric morphing sequence of the Stanford Bunny model (side view) using the method in [42]. $t$ is the time parameter in the morphing process.
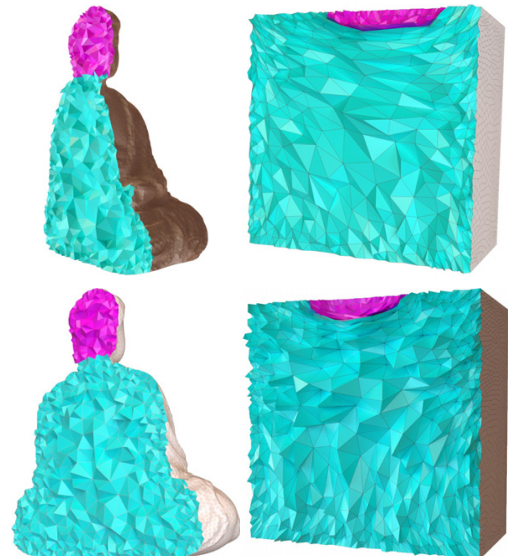

(a) (b) $S=0.25$
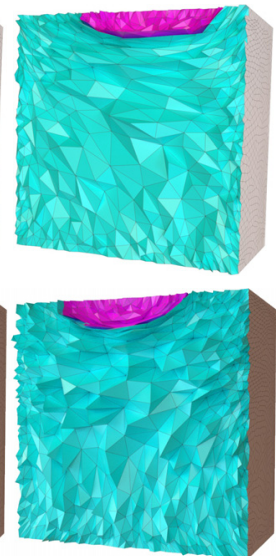

(c) $S=0.5$
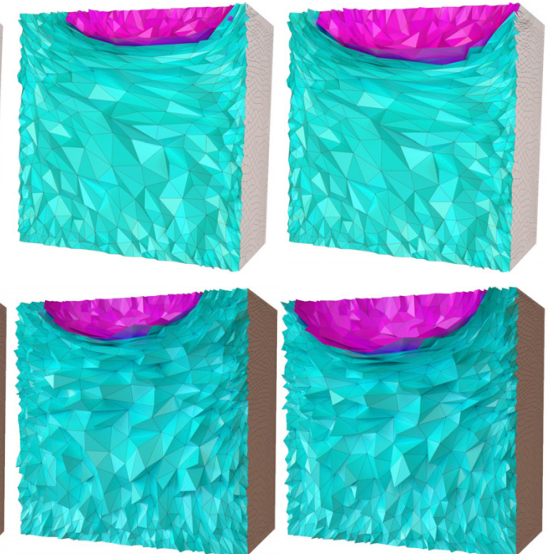

(d) $S=1$

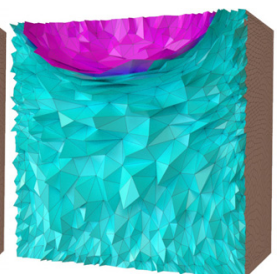

(e) $S=2$

Figure 7: ROI of the Buddha Model and the importance driven volume parameterization. (a) the Buddha volumetric mesh; (b)-(e) show importance driven results of ROIs with different scaling factors. The top and bottom rows show the solid meshes from different views. 

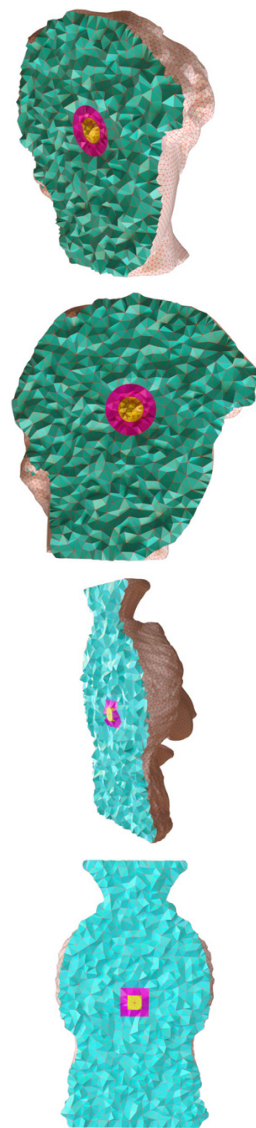

(a)
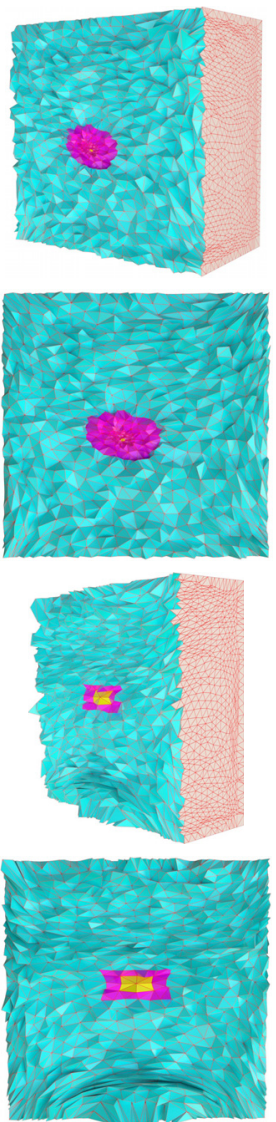

(b) $S=1$
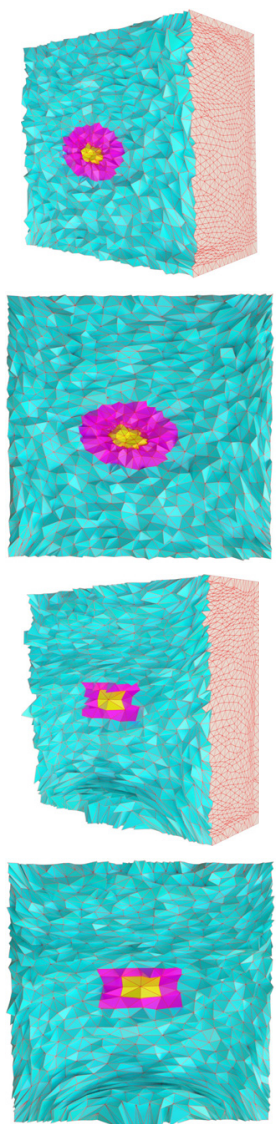

(c) $S=2$
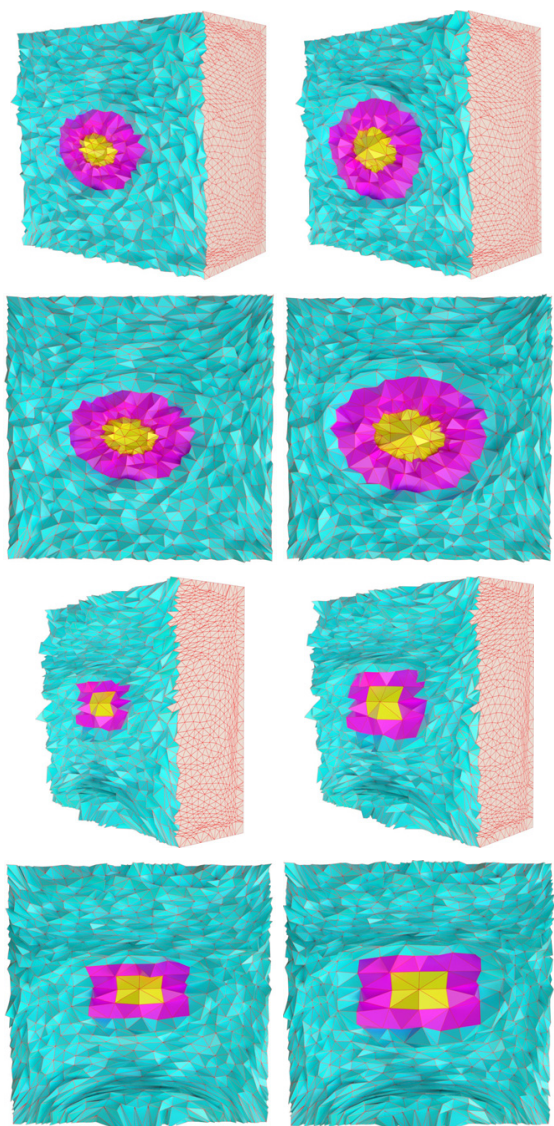

(d) $S=4$
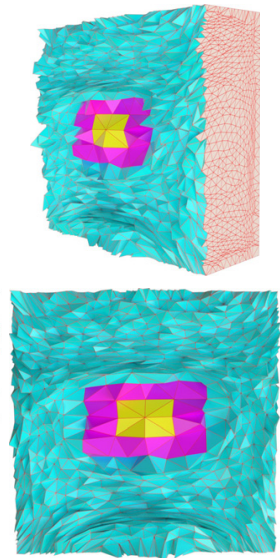

(e) $S=8$

Figure 8: The multiple ROIs of the David King and Lion Cup model and the importance driven volume parameterization. Column (a) is the David Head and Lion Cup volumetric mesh; (b)-(e) shows importance driven results of ROIs with different scale factors. The ROI of the David Head is two solid balls while that of the Lion Cup is two solid cubes.

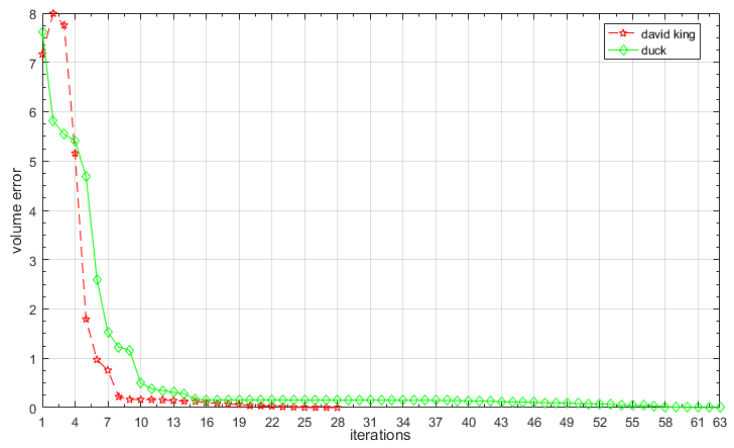

Figure 9: Convergence curves of the Newton's method.

We report the number of flipped tetrahedra in the table as well. The mesh quality of the tetrahedral mesh affects the harmonic mapping, especially the number of flipped tetrahedra. If many dihedral angles are obtuse, then the harmonic map will induce more flipped tetrahedra.

Fig.9 shows the convergence curves of our method on the Bimba model and the King David's head model. The horizontal axis is the number of iterations, the vertical axis is the $L^{2}$ nor$\mathrm{m}$ of the target measure and the current measure. The curves demonstrate the fast convergence rate of our algorithm.

\section{Summary and Future Directions}

This work proposes a novel volumetric parameterization method with user prescribed measures for simply connected tetrahedron mesh with a single boundary surface. Our method is based on the optimal mess transportation theory, that is also capable of handling solids with voids inside. The algorithm includes two main steps, volumetric harmonic mapping, and volumetric optimal mass transportation map.

The method has solid theoretic foundation, utilizing the mature algorithms from computational geometry (Power voronoi diagram), and producing volumetric parameterizations with the measure controllability. We have tested our algorithm on many models in real life, which demonstrates the efficiency and efficacy of the method.

In practice, the volumetric harmonic map may produce mappings with flipped tetrahedra for meshes with complicated geometries and low quality tetrahedron meshing. One sufficien$\mathrm{t}$ condition to guarantee the homeomorphism is that the input solid is star-shaped as described in [9]. We will explore other sufficient conditions. In fact, the first step, volumetric harmonic map, can be replaced by any algorithm that can produce a homeomorphism from the input solid to a convex domain in the Euclidean space, such as the generalized quasi-conformal map- 
(a)
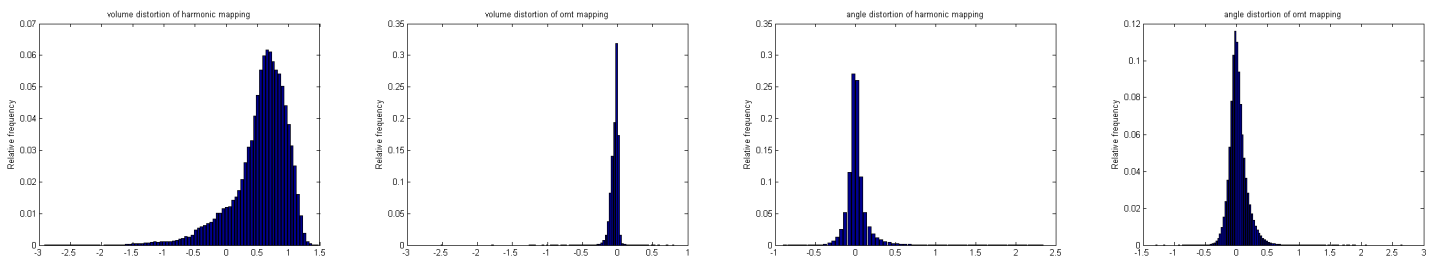

(b)
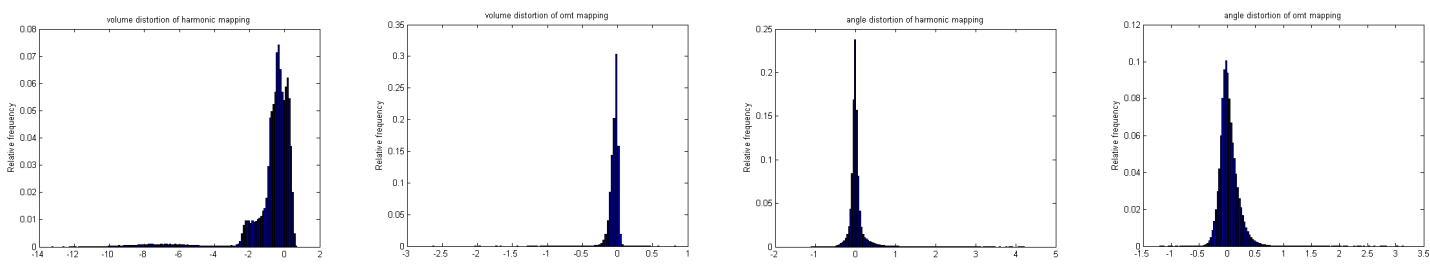

(c)
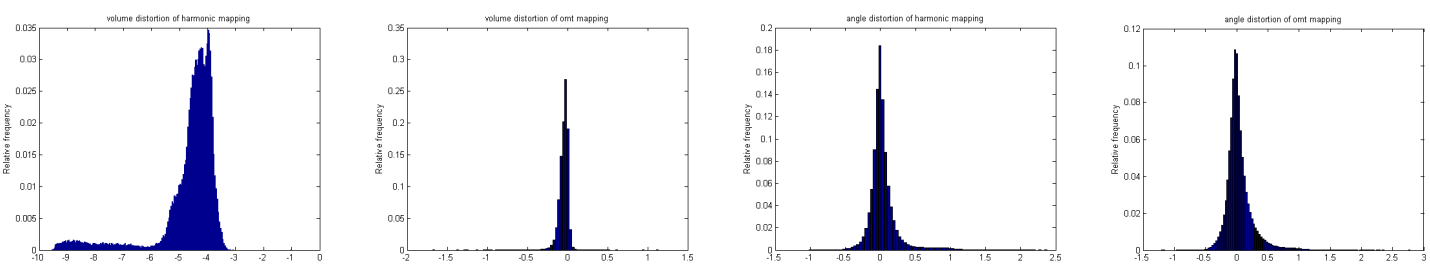

(d)
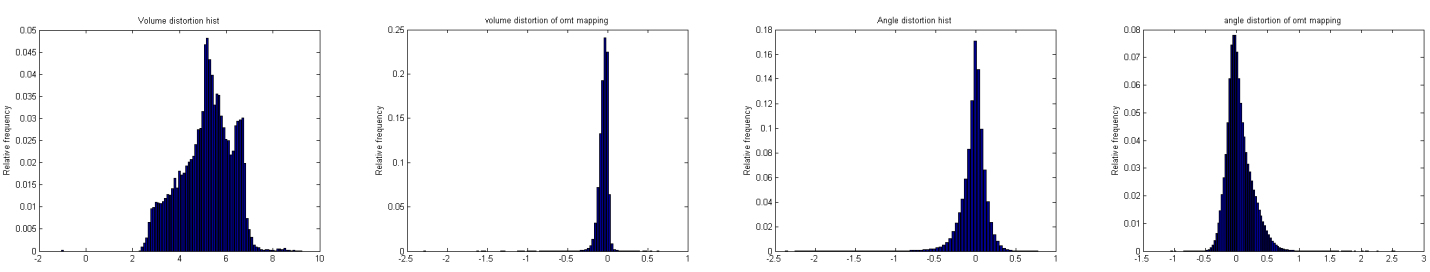

(e)
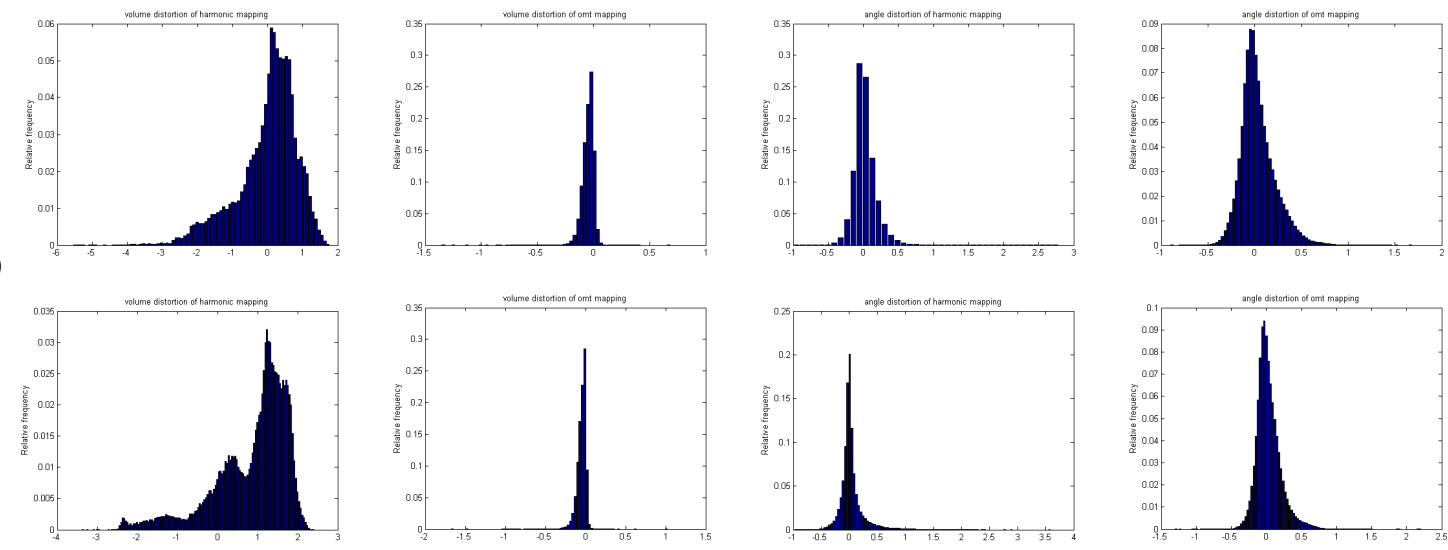

(1)

(2)

(3)

(4)

Figure 10: The histograms of the volume and angle distortion. Row (a) to (f) show the distortion histograms of the King David's Head, the Stanford Bunny, the Buddha, the Bimba, the Duck and the Lion Cup model. Column (1) and (2) show the volume distortion of our method is much lower than others. Column (3) and (4) illustrate the angle distortion of our method.

Table 1: Performance Statistics

\begin{tabular}{|c|c|c|c|c|c|c|c|}
\hline Model & Vertices & Tetrahedra & \multicolumn{2}{|c|}{ Number of Iterations } & \multicolumn{2}{|c|}{ Time in Second } & Flipped \\
\cline { 4 - 8 } & & & Our Method & Gradient Descent & Newton's Method & Gradient Descent & Tetrahedra \\
\hline David King & 19984 & 90576 & 28 & 1536 & 586 & 32013 & 0 \\
\hline Bunny & 22466 & 116740 & 446 & 3285 & 12220 & 84136 & 15 \\
\hline Buddha & 20934 & 99113 & 110 & 2660 & 3570 & 74028 & 6 \\
\hline Duck & 20320 & 96873 & 63 & 1852 & 2049 & 60179 & 7 \\
\hline Bimba & 21700 & 89282 & 99 & 2623 & 3025 & 70875 & 8 \\
\hline Lion-cup & 20874 & 97132 & 59 & 1844 & 2149 & 64254 & 5 \\
\hline
\end{tabular}


ping [43]. In the future, we will explore alternative volumetric mapping to replace the volumetric harmonic mapping step. Furthermore, we will generalize the method to volumes with more complicated topologies.

\section{Acknowledgement}

The authors thank all the anonymous reviewers, their comments and critiques are extremely valuable to improve the quality of the current work. The project is partially supported by NSF DMS-1418255, AFOSR FA9550-14-1-0193, NSFC 11271156, NSFC 11001017.

\section{References}

[1] J. A. Cottrell, T. J. R. Hughes, Y. Bazilevs, Isogeometric Analysis: Toward Integration of CAD and FEA, Wiley, 2009.

[2] H. Si, Tetgen, a delaunay-based tetrahedral mesh generator, ACM Transactions on Mathematical Software 41 (2) (2015) 11:1-11:36.

[3] Y. Wang, X. Gu, S.-T. Yau, Volumetric harmonic map, Communications in Information and Systems 3 (3) (2004) 191-202.

[4] X. Gu, F. Luo, J. Sun, S.-T. Yau, Variational principles for Minkowski type problems, discrete optimal transport, and discrete Monge-Amperé equations, Asian Journal of Mathematics (AJM) 20 (2) (2016) 383-398.

[5] Y. Wang, X. Gu, T. F. Chan, P. M. Thompson, S.-T. Yau, Brain surface conformal mapping and brain volumetric harmonic map with variational methods, in: SIAM Conference on Imaging Science, 2004.

[6] X. Li, X. Guo, H. Wang, Y. He, X. Gu, H. Qin, Meshless harmonic volumetric mapping using fundamental solution methods, IEEE Transactions on Automation Science and Engineering 6 (3) (2009) 409-422.

[7] T. Marin, E. Cohan, R. M. Kirby, Volumetric parameteriztion and trivariate b-spline fitting using harmonic function, Computer Aided Geometric Design 26 (6) (2009) 648-664.

[8] H. Xu, W. Yu, S. Gu, X. Li, Biharmonic volumetric mapping using fundamental solutions, IEEE Transactions on Visualization and Computer Graphics 19 (5) (2013) 787-798.

[9] J. Xia, Y. He, S. Han, C. W. Fu, F. Luo, X. Gu, Parameterization of starshaped volumes using green's functions, in: Advances in Geometric Modeling and Processing, Vol. 6130, Springer, 2010, pp. 219-235.

[10] J. Xia, Y. He, X. Yin, S. Han, X. Gu, Direct-product volumetric parameterization of handlebodies via harmonic fields, in: Shape Modeling International (SMI), 2010.

[11] V. Gupta, H. K. Voruganti, B. Dasgupta, Domain mapping for volumetric parameterization using harmonic functions, Computer-Aided Design and Applications 11 (4) (2014) 426-435.

[12] X. Y. Li, T. Ju, S. M. Hu, Cubic mean value coordinates, ACM Transactions on Graphics 32 (4) (2013) 126:1-126:10.

[13] M. S. Floater, Mean value coordinates, Computer Aided Geometric Design 20 (1) (2003) 19-27.

[14] T. Ju, S. Schaefer, J. Warren, Mean value coordinates for closed triangular meshes, ACM Transactions on Graphics 24 (3) (2005) 561-566.

[15] G. K. M. S. Floater, M. Reimers, Mean value coordinates in 3d, Computer Aided Geometric Design 22 (7) (2005) 623-631.

[16] Y. Lipman, D. Levin, D. Cohen-Or, Green coordinates, ACM Transactions on Graphics 27 (3) (2008) 78:1-78:10.

[17] I. Chao, U. Pinkall, P. Sanan, P. Schröder, A simple geometric model for elastic deformations, ACM TOG 29 (4) (2010) 38:1-38:6.

[18] M. Nieser, U. Reitebuch, K. Polthier, Cube cover parameterization of $3 \mathrm{~d}$ volumes, Computer Graphics Forum 30 (5) (2011) 1397-1406.

[19] J. Huang, Y. Tong, H. Wei, H. Bao, Boundary aligned smooth 3d crossframe field, ACM Transactions on Graphics 30 (6) (2011) 143:1-143:8.

[20] Y. Li, Y. Liu, W. Xu, W. Wang, B. Guo, All-hex meshing using singularity-restricted field, ACM TOG 31 (6) (2012) 177:1-177:11.

[21] Y. Jin, G.-P. Qian, J.-Y. Zhao, J. Chang, R.-F. Tong, J. Zhang, Stretchminimizing volumetric parameterization, Journal of computer science and technology 30 (3) (2015) 553-564.

[22] N. Bonnotte, From Knothe's rearrangement to Brenier's optimal transport map, arXiv:1205.1099 (2012) 1-29.

[23] L. V. Kantorovich, On a problem of Monge, Uspekhi Mat. Nauk. 3 (1948) 225-226.
[24] S. Rachev, L. Ruschendorf, Mass Transportation Problems, Vol. I-II, Springer, New York, 1998.

[25] A. Dominitz, A. Tannenbaum, Texture mapping via optimal mass transport, TVCG 16 (13) (2010) 419-432.

[26] T.Rehman, E.Haber, G.Pryor, J.Melonakos, A.Tannenbaum, 3D nonrigid registration via optimal mass transport on the GPU, Med. Image Analy. 13 (2009) 931-40.

[27] Y. Brenier, Polar factorization and monotone rearrangement of vectorvalued functions, Com. Pure Appl. Math. 64 (1991) 375-417.

[28] Z. Su, Y. Wang, R. Shi, W. Zeng, J. Sun, F. Luo, X. Gu, Optimal mass transport for shape matching and comparison, TPAMI 37 (11) (2015) 2246-2259.

[29] X. Zhao, Z. Su, X. D. Gu, A. Kaufman, J. Sun, J. Gao, F. Luo, Area-preservation mapping using optimal mass transport, TVCG 19 (12) (2013) 2838-2847.

[30] C. Villani, Topics in optimal transportation, no. 58 in Graduate Studies in Mathematics, American Mathematical Soc., 2003.

[31] C. Villani, Topics in Optimal Transportation, American Mathematical Society, 2003.

[32] A. D. Alexandrov, Convex Polyhedra, Springer, 2005.

[33] A. V. Pogorelov, The regularity of the generalized solutions of the equation $\operatorname{det}\left(\partial^{2} u / \partial x^{i} \partial x^{j}\right)=\phi\left(x^{1}, x^{2}, \ldots, x^{n}\right) \dot{0}($ (russian), Dokl. Akad. Nauk SSSR 200 (1971) 534-537.

[34] F. Aurenhammer, Power diagrams: properties, algorithms and applications, SIAM J. Comput. 16 (1987) 78-96.

[35] F. Aurenhammer, F. Hoffmann, B. Aronov, Minkowski-type theorems and least-squares clustering, Algorithmica 20 (1) (1998) 61-76.

[36] Q. Mérigot, E. Oudet, Discrete optimal transport: complexity, geometry and applications, Discrete \& Computational Geometry (2014) 1-21.

[37] Q. Merigot, A multiscale approach to optimal transport, Computer Graphics Forum 30 (5) (2011) 1583-1592.

[38] M. D. Berg, M. V. Kreveld, M. Overmars, O. C. Schwarzkopf, Computational Geometry, Springer, 2000.

[39] G. Guennebaud, B. Jacob, Eigen v3, http://eigen.tuxfamily.org (2010).

[40] J. Kitagawa, Q. Mérigot, B. Thibert.

[41] T. C. Project, CGAL User and Reference Manual, CGAL Editorial Board, 2015.

URL http: //doc.cgal .org/4.7/Manual/packages.html

[42] B. Lévy, A numerical algorithm for $l^{2}$ semi-discrete optimal transpot in 3d., ESAIM: Mathematical Modelling \& Numerical Analysis 49 (6) (2015) 1693 - 1715 .

[43] Y. T. Lee, K. C. Lam, L. M. Lui, Landmark-matching transformation with large deformation via n-dimensional quasi-conformal maps, Journal of Scientific Computing (2015) 1-29. 